\title{
Structure effect on one neutron removal reaction using relativistic mean field densities in Glauber model
}

\author{
R. N. Panda ${ }^{1}$, and S. K. Patra ${ }^{2}$ \\ ${ }^{1}$ Department of Physics, ITER, Siksha 'O' Anusandhan University, Bhubaneswar-751 030, India, \\ ${ }^{2}$ Institute of Physics, Sachivalaya Marg, Bhubaneswar-751 005, India.
}

(Dated: January 3, 2019)

\begin{abstract}
We calculate the one neutron removal reaction cross-section $\left(\sigma_{-1 n}\right)$ for few stable and neutron-rich halo nuclei with ${ }^{12} \mathrm{C}$ as target, using relativistic mean field (RMF) densities, in the frame work of Glauber model. The results are compared with the experimental data. Study of the stable nuclei with the deformed densities have shown a good agreement with the data, however, it differs significantly for the halo nuclei. We observe that while estimating the $\sigma_{-1 n}$ value from the difference of reaction cross-section of two neighboring nuclei with mass number A and that of A-1 in an isotopic chain, we get good agreement with the known experimental data for the halo cases.

PACS numbers: 21.10.Gv, 24.10.-i, 25.40.-h, 24.10.Jv
\end{abstract}

\section{INTRODUCTION}

From last two decades or more, the exploration of neutronrich nuclei is an important branch in Nuclear Physics research. It is a source of observance of new phenomena and dynamics. This is possible due to the development of accelerator techniques for Radioactive Ion Beams (RIBs) in various laboratories around the globe. Experimental methods and theoretical analysis have been widely used to collect information about the structure information, such as nuclear size, valence nucleon distribution and halo structure of these exotic nuclei. The measurement of various reaction observables like total reaction cross-section $\sigma_{r}$, one- and two- nucleon removal crosssection $\left(\sigma_{-1 n}, \sigma_{-2 n}\right)$ and the longitudinal momentum distribution $P_{\|}$are some of the established quantities for such studies.

The relativistic mean field (RMF) or the effective field theory motivated RMF, i.e., the E-RMF provides the internal structure or sub-structure information of the nuclei through the density distributions, using as an input while calculating the observables in conjunction with Glauber model [1, 2]. A systematic study of one- and two-neutron knockout data for ${ }^{15-19} \mathrm{C}$, explained beautifully while using shell model, which gives a consistent structure information not only for the stable nuclei but also neutrons at the boundary [3]. It is well known that narrow fragment momentum distribution reflects large space distribution of the valence nucleon and there is a correlation of the magnitude of the $\sigma_{-1 n}$ with the width of the $P_{\|}$, in approaching the nucleon (neutron or proton) driplines. However, one-neutron removal reaction cross-sections provide important nuclear structure information complementary to that obtained from $P_{\|}$.

In the present paper, our aim is to calculate the $\sigma_{r}$ and $\sigma_{-1 n}$ by using densities obtained from the RMF and E-RMF formalisms [1, 2] in conjunction with Glauber model. It will be shown for the nuclei near the drip-line which possess a halostructure fails to be explained by the standard evaluation of $\sigma_{-1 n}$. Contrary to this estimation, the difference in total reaction cross-section between two consecutive neighboring nuclei in an isotopic chain better matches with the experimental data.

\section{THEORETICAL FRAMEWORK}

The use of RMF and E-RMF formalisms for finite nuclei as well as infinite nuclear matter are well documented and details can be found in [4, 5] and [6, 7] respectively. The working expressions for density profiles and other related quantities are available in [1, 2, 4, 6, 7]. The details to calculate $\sigma_{r}$ using Glauber approach has been given by R. J. Glauber [8]. This model is based on the independent, individual nucleonnucleon $(N N)$ collisions in the overlapping zone of the colliding nuclei, and has been used extensively to explain the observed total nuclear reaction cross-sections for various systems at high energies. The standard Glauber form for the total reaction cross-sections at high energies is expressed as [8, 9]:

$$
\sigma_{r}=2 \pi \int_{0}^{\infty} b[1-T(b)] d b,
$$

where $T(b)$ is the transparency function with impact parameter $b$. The function $T(b)$ is calculated in the overlap region between the projectile and the target assuming the interaction is formed from a single $N N$ collision. It is given by

$$
T(b)=\exp \left[-\sum_{i, j} \bar{\sigma}_{i j} \int d \bar{s}_{t i}(s) \bar{\rho}_{p j}(|\vec{b}-\vec{s}| s)\right] .
$$

The summation indices $i$ and $j$ run over proton and nucleon and subscripts $p$ and $t$ referred to projectile and target, respectively. The experimental nucleon-nucleon reaction crosssection $\bar{\sigma}_{i j}$ varies with energy. The $z$-integrated densities $\bar{\rho}(\omega)$ are defined as

$$
\bar{\rho}(\omega)=\int_{-\infty}^{\infty} \rho\left(\sqrt{\omega^{2}+z^{2}}\right) d z
$$

with $\omega^{2}=x^{2}+y^{2}$. The argument of $T(b)$ in Eq. (2) is $|\vec{b}-\vec{s}|$, which stands for the impact parameter between the $i^{\text {th }}$ and $j^{\text {th }}$ nucleons. 
The original Glauber model was designed for high energy approximation. However, it was found to work reasonably well for both the nucleus-nucleus reaction and the differential elastic scattering cross-sections over a broad energy range [10]. To include the low energy effects of $N N$ interaction, the Glauber model is modified to take care of the finite range effects in the profile function and Coulomb modified trajectories [11, 12]. The modified $T(b)$ is given by [12, 13],

$$
T(b)=\exp \left[-\int_{p} \int_{t} \sum_{i, j}\left[\Gamma_{i j}(\vec{b}-\vec{s}+\vec{t})\right] \bar{\rho}_{p i}(\vec{t}) \bar{\rho}_{t j}(\vec{s}) d \vec{s} d \vec{t}\right] .
$$

The profile function $\Gamma_{i j}\left(b_{e f f}\right)$ is defined as [1]

$$
\Gamma_{i j}\left(b_{e f f}\right)=\frac{1-i \alpha_{N N}}{2 \pi \beta_{N N}^{2}} \sigma_{i j} \exp \left(-\frac{b_{e f f}^{2}}{2 \beta_{N N}^{2}}\right)
$$

with $b_{\text {eff }}=|\vec{b}-\vec{s}+\vec{t}|, \vec{s}$ and $\vec{t}$ are the dummy variables for integration over the $z$-integrated target and projectile densities. The parameters $\sigma_{N N}, \alpha_{N N}$, and $\beta_{N N}$ are usually case-dependent (proton-proton, enutron-neutron or protonneutron), but we have used the appropriate average values from Refs. [9, 14]. The deformed or spherical nuclear densities obtained from the RMF and E-RMF models are fitted to a sum of two Gaussian functions with suitable co-efficients $c_{i}$ and ranges $a_{i}$ chosen for the respective nuclei which is expressed as

$$
\rho(r)=\sum_{i=1}^{2} c_{i} \exp \left[-a_{i} r^{2}\right]
$$

Then, the Glauber model is used to calculate the total reaction cross-section for both the stable and unstable nuclei considered in the present study.

The expression for one nucleon removal reaction crosssection $\sigma_{-1 n}(I)$ is given by [12]

$$
\sigma_{-1 n}(I)=\sum_{c} \int d \vec{k} \sigma_{a=(k, g=0), c}
$$

where $\sigma_{a=(k, g=0), c}$ are the possible final states $a c$. In the present formalism, it is considered that the projectile nucleus breaks up into a core and the removed nucleon. The core $C$ has an internal wave function $\phi_{g}$ and the one-nucleon, i.e., the departed nucleon has an asymptotic momentum $\hbar \mathbf{k}$ in the continuum state with respect to the core. The core is considered to be in the ground state $(g=0)$ at the time of the collision. The total $\sigma_{-1 n}(I)$ can be separated to an elastic $\left(\sigma_{-1 n}^{e l}\right)$ with $c=0$ and inelastic $\left(\sigma_{-1 n}^{i e l}\right)$ part having $c$ as non-zero. The $\sigma_{-1 n}^{e l}$ and $\sigma_{-1 n}^{i e l}$ is expressed as [12]

$$
\begin{aligned}
\sigma_{-1 n}^{e l}(I)= & \int d \mathbf{b}\left\{<\phi_{0}\left|e^{-2 \operatorname{Im} \chi_{C t}\left(b_{C}\right)-2 \operatorname{Im} \chi_{-1 n t}\left(b_{C}+s\right)}\right|\right. \\
& \left.-\left|<\phi_{0}\right| e^{-i \chi_{C t}\left(b_{C}\right)+i \chi_{-1 n t}\left(b_{C}+s\right)}\left|\phi_{0}>\right|^{2}\right\}
\end{aligned}
$$

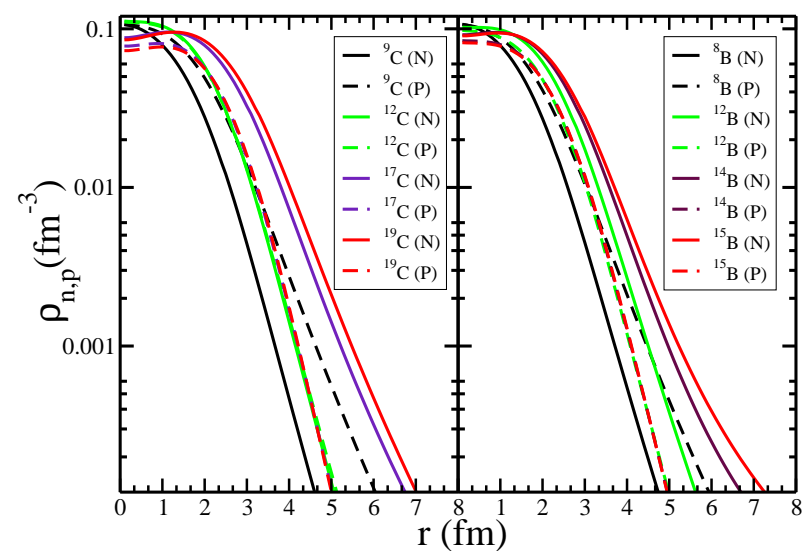

FIG. 1: The spherical proton $\left(\rho_{p}\right)$ and neutron $\left(\rho_{n}\right)$ density obtained from RMF (NL3) parameter set for various isotopes of (a) Carbon and (b) Boron.

$$
\begin{aligned}
\sigma_{-1 n}^{i e l}(I)= & \int d \mathbf{b}\left\{<\phi_{0} \mid e^{-2 \operatorname{Im} \chi_{C t}\left(b_{C}\right)}\right. \\
& \left.-e^{-2 \operatorname{Im} \chi_{C t}\left(b_{C}\right)-2 \operatorname{Im} \chi_{-1 n t}\left(b_{C}+s\right)} \mid \phi_{0}>\right\}
\end{aligned}
$$

here $\chi_{p t}$ is the phase shift function and $\phi_{0}$ is the valence wave function (the wavefunction of the removed nucleon). The notation and the numerical procedure of calculation of onenucleon removal reaction cross-section are followed from Ref. [12].

\section{CALCULATIONS AND RESULTS}

We obtain the field equations for nucleons and mesons from the RMF and E-RMF lagrangian. For deformed case (RMF only), these equations are solved by expanding the upper and lower components of the Dirac spinners and the boson fields in an axially deformed harmonic oscillator basis. The set of coupled equations are solved numerically by a self-consistent iteration method taking different inputs of the initial deformation $\beta_{0}$ [4, 15]. For spherical densities both for RMF and E-RMF models, we follow the numerical procedure of Refs. [7]. In our calculation, the constant gap BCS pairing is used to add the pairing effects for open shell nuclei. The centreof-mass motion (c.m.) energy correction is estimated by the usual harmonic oscillator formula $E_{\text {c.m. }}=\frac{3}{4}\left(41 A^{-1 / 3}\right)$.

Comparing the binding energy (BE) of the calculated solutions, the maximum $\mathrm{BE}$ and the corresponding densities $\left[\rho_{p}\right.$ (proton) and $\rho_{n}$ (neutron)] are for the ground state. All other solutions are the excited intrinsic state including the spherical one. Since the main input in the Glauber model estimation is the RMF or E-RMF densities, it is important to have an information of these quantities. We have plotted the spherical $\phi_{0}>\rho_{p}$ and $\rho_{n}$ for both proton and neutron of Carbon and Boron isotopes in Figure 1 using RMF (NL3) parameter set [5]. As (8)xpected, we find an extended density distribution for proton 
compared to neutron in case of ${ }^{9} \mathrm{C}$ and ${ }^{8} \mathrm{~B}$ due to the protonrich nature of these two nuclei. The value of $\rho_{n}$ and $\rho_{p}$ are almost similar for ${ }^{12} \mathrm{C}$ which can be seen from Figure 1. Extension of $\rho_{n}$ is much more than $\rho_{p}$ for rest of the nuclei. It is maximum for ${ }^{19} \mathrm{C}$ and ${ }^{15} \mathrm{~B}$ in Carbon and Boron isotopic chains, respectively, because of the high neutron to proton ratio for these cases.

In the present study of $\sigma_{r}$ and $\sigma_{-1 n}$, first we use the spherical density obtained from RMF (NL3)[5] and E-RMF (G2) [6]. The results are presented in Table 1 for ${ }^{9,12,13,15,17,19} \mathrm{C}$ and ${ }^{8,12,13,14,15} B$ isotopes with ${ }^{12} C$-target at various projectile energies. These results deviate considerably from the data [16-19] which are quoted in the table. For example, in case of ${ }^{9} \mathrm{C}+{ }^{12} \mathrm{C}$, the observed value of $\sigma_{-1 n}$ is $48 \pm 8 \mathrm{mb}$ as compared to the estimated results of 81 and $96 \mathrm{mb}$ with NL3 and G2 parametrization, respectively. Note that the $\sigma_{1 n}$ for ${ }^{8} B+{ }^{12} C$ and ${ }^{9} C+{ }^{1} 2 C$ systems are one-proton removal reaction cross-section, which may be followed throughout the text and Tables. However, in rest of the systems, $\sigma_{1 n}$ will be refered as one-neutron removal reaction cross-section. Similar discrepancy is also seen for other cases. A further inspection of the table shows that the experimental one-neutron removal reaction cross-section for some selected cases coincide well with the prediction. We also used the method of B. Abu-Ibrahim et al. [12] to calculate the one-neutron removal reaction cross-section $\sigma_{-1 n}(I I)$, which obtain by the difference of total reaction cross-section of two neighboring nuclei with mass number A and A-1 in an isotopic chain. This prescription is suitable only for halo projectile and may not be applicable for general cases. The expression is given by [12]:

$$
\sigma_{-1 n}(I I)=\sigma_{r}\left({ }^{A} Z\right)-\sigma_{r}\left({ }^{A-1} Z\right),
$$

and the values are inserted in Table 1 for comparison. The $\sigma_{-1 n}(I I)$ differs significantly from the experimental data for all the cases. It is important to recall that the effect of deformation is nominal in the evaluation of $\sigma_{r}$ which is reported in our earlier publications [1, 2]. In these papers, the Glauber model with RMF (NL3, NL-SH) and E-RMF (G2) densities show a good agreement with experimental data for $\sigma_{r}$ and elastic differential scattering cross-sections $d \sigma / d \Omega$, which in general justify the model independency of the calculation with various relativistic parametrizations.

Unlike to the total reaction cross-section, the $\sigma_{-1 n}$ obtained from the Glauber model, depends very much on the structure information of the projectile and target nuclei, i.e., input densities of these systems.

The nuclear single-particle energy $\epsilon_{n, p}$ for the last occupied orbit is very important for a reaction process. Thus, it is worthwhile to analyse the $\epsilon_{n, p}$ of the valence nucleon of the projectile and target nuclei. For simplicity, the spherical single-particle energy for the last occupied orbit for proton $\epsilon_{p}$ and neutron $\epsilon_{n}$ with RMF (NL3) and E-RMF (G2) are compared. As expected, a small variant in $\epsilon_{p}$ or $\epsilon_{n}$ makes a remarkable change in $\sigma_{-1 n}(I I)$ [Eq. (10)] for many cases. For example, the one neutron removal reaction cross-section $\sigma_{-1 n}(I I)$, for ${ }^{12} C+{ }^{12} C$ are 55 and $39 \mathrm{mb}$ for RMF (NL3) and E-RMF (G2) with their single-particle energies $\epsilon_{p}=$ $-15.66, \epsilon_{n}=-18.97$ and $\epsilon_{p}=-13.16, \epsilon_{n}=-16.16 \mathrm{MeV}$, respectively. This discrepancy is minimum in the calculation of $\sigma_{-1 n}(I)$ [Eq. (7)] with a lone exception for ${ }^{13} B+{ }^{12} C$ system. As the valence $\epsilon_{n, p}$ plays a major role to determine the reaction observables, one needs to reproduce these values with the experimental observation. This can be achieved by a small adjustment of the parameters in the relativistic mean field formalisms. However, the philosophy of RMF or E-RMF of single set of parametrization for the entire domain of nuclear landscape goes against this parameter fiddling. Keeping this in mind, the quality of the results is compromised slightly using with the original values of NL3, NL-SH or G2 sets.

Apart from the single-particle energy, the structure effect of the participating nuclei is crucial for a reaction study. In this context, it is interesting enough to see this effect (deformation effect) on $\sigma_{-1 n}$. We repeat the calculations for $\sigma_{-1 n}(I)$ and $\sigma_{-1 n}(I I)$ with the deformed densities (RMF only) as input in the Glauber model [1]. We obtain spherical equivalent of the axially deformed densities using equations (3) and (6) following the prescription of Refs. [1, 2]. The NL-SH parameter set [20] for this purpose is used and the results are listed in Table 2. The reason to change the NL3 to NL-SH is the unavailability of converged ground state deformed solution with NL3 for very light mass nuclei, which is a situation in the present study [21]. Also, the NL-SH parametrization is reasonably a better parameter set and we expect similar outcome from this force parameter.

Due to similar reason as mentioned for the spherical nuclei, the deformed densities for some selected cases are imperative to analyse. Our earlier work on density study supply us enough signature about the complicated sub-structure [22]. The clustering and sub-structure of these deformed neutron and proton density distributions are demonstrated in Figure 2. The density contours presented are in boxes of width and height $6 \mathrm{fm}$. A uniform contour spacing of $0.01 \mathrm{fm}^{-3}$ is used for proton and neutron densities. The z-axis is chosen as the symmetry axis, the densities are evaluated in the $z \rho$ plane, where $x=y=\rho$. In ref. [22], it is noticed that ${ }^{12} \mathrm{C}$ possesses a $3 \alpha$-cluster with a tetrahedral configuration. The same structure is reproduced in the present study with an oblate shape. The structure of the neutron deficient ${ }^{9} \mathrm{C}$ nucleus has a prolate ground state and that of the neutron-rich ${ }^{19} \mathrm{C}$ has an oblate ground state deformation. In all the three cases, the density plot informs that the central part of the nucleus is a compact core, which is surrounded by a thin layer of nucleons. The structure of the internal core for both proton and neutron have different density distribution from ${ }^{9} \mathrm{C}$ to ${ }^{19} \mathrm{C}$. The shape of ${ }^{19} \mathrm{C}$ proton density distribution looks like a perfect dumb-bell. Thus, it has a maximum probability to to have the structural effects on neutron removal reaction. On the other hand, the total nuclear reaction cross-section is less influenced by deformation, may be because of the averaging in input density in the Glauber model calculations.

The results obtained from the deformed densities are tabulated (see Table 2). Table 2 shows that most of the $\sigma_{-1 n}(I)$ [obtained from Eq. (7)] matches quite well with the experimental data of [16-19] and few of them do not agree. On the other hand $\sigma_{-1 n}$ evaluated from Eq. (10) coincide with only ${ }^{19} C+{ }^{12} C$ experimental data [16]. Of particular interest 
TABLE I: One-neutron- for ${ }^{12,13,15,17,19} C$ and one-proton removal reaction cross-sections $\sigma_{-1 n}$ (in mb) for ${ }^{9} C$ and ${ }^{8} \mathrm{~B}$ with ${ }^{12} C-$ target using spherical RMF and E-RMF densities obtained from NL3 and G2 parameter sets, respectively. The available experimental data [16-19] is given for the comparison. The last occupied proton and neutron single-particle energies are also given.

\begin{tabular}{|c|c|c|c|c|c|c|c|c|c|c|}
\hline \hline Projectile & Energy & $\begin{array}{c}\sigma_{-1 n} \\
\text { Exp. }\end{array}$ & \multicolumn{2}{c|}{$\sigma_{-1 n}(I)$} & \multicolumn{2}{c|}{$\sigma_{-1 n}(I I)$} & \multicolumn{2}{c|}{$\epsilon_{p}(M e V)$} & \multicolumn{2}{c|}{$\epsilon_{n}(M e V)$} \\
& & RMF & E-RMF & RMF & E-RMF & RMF & E-RMF & RMF & E-RMF \\
\hline${ }^{9} C$ & 285 & $48(8)$ & 81 & 96 & 34 & 31 & -4.07 & -4.15 & -13.81 & -13.90 \\
${ }^{12} C$ & 1050 & $44.7(3)$ & 39 & 37 & 55 & 39 & -15.66 & -13.16 & -18.97 & -16.16 \\
${ }^{13} C$ & 800 & & 29 & 28 & 47 & 37 & -16.81 & -15.30 & -17.77 & -16.35 \\
${ }^{15} C$ & 54 & $137(16)$ & 95 & 88 & 37 & 38 & -19.18 & -20.47 & -10.57 & -10.39 \\
${ }^{17} C$ & 904 & $129(22)$ & 101 & 95 & 45 & 32 & -23.93 & -22.65 & -11.53 & -11.57 \\
${ }^{19} C$ & 910 & $231(51)$ & 134 & 128 & 35 & 37 & -27.25 & -25.89 & -12.61 & -12.50 \\
${ }^{8} B$ & 285 & $89(2)$ & 103 & 107 & 47 & 33 & -23.16 & -20.73 & -31.50 & -28.96 \\
${ }^{12} B$ & 67 & $81(5)$ & 54 & 49 & 47 & 44 & -39.95 & -34.18 & -14.20 & -13.49 \\
${ }^{13} B$ & 57 & $59(4)$ & 54 & 38 & 46 & 43 & -39.88 & -35.49 & -7.81 & -7.85 \\
${ }^{14} B$ & 50 & $153(15)$ & 70 & 64 & 48 & 38 & -41.00 & -36.85 & -8.40 & -8.41 \\
${ }^{15} B$ & 43 & $108(13)$ & 87 & 89 & 51 & 39 & -42.16 & -38.23 & -8.77 & -8.96 \\
\hline \hline
\end{tabular}

TABLE II: Same as Table 1, but using both spherical and deformed NL-SH densities for ${ }^{9,12,15,17,19} C$ and ${ }^{8,12,13,14,15} B$ projectiles taking ${ }^{12} C$ as target. The available experimental data is also displayed for the comparison [16-19]. The last occupied neutron and proton single-particle orbits are also given and it is denoted by the Nilsson index $\left[N n_{3} \Lambda\right] \Omega^{\pi}$.

\begin{tabular}{|c|c|c|c|c|c|c|c|c|c|c|c|c|}
\hline \hline Projectile & Target & Energy & $\begin{array}{c}\sigma_{-1 n} \\
\text { Exp. }\end{array}$ & \multicolumn{2}{c|}{$\sigma_{-1 n}(I)$} & \multicolumn{2}{c|}{$\sigma_{-1 n}(I I)$} & $\beta_{2}$ & \multicolumn{2}{|c|}{ neutron } & \multicolumn{2}{|c|}{ proton } \\
& & & Sph. & Sph. & Def. & & {$\left[N n_{3} \Lambda\right]$} & $\epsilon_{n}(\mathrm{MeV})$ & {$\left[\mathrm{Nn} n_{3} \Lambda\right]$} & $\epsilon_{p}(\mathrm{MeV})$ \\
\hline${ }^{9} C$ & ${ }^{12} C$ & 285 & $48(8)$ & 70 & 66 & 24 & 03 & 0.36 & {$[110] 1^{-}$} & -16.122 & {$[101] 3^{-}$} & -2.772 \\
${ }^{12} C$ & ${ }^{12} C$ & 1050 & $44.7(3)$ & 39 & 49 & 45 & 293 & -0.21 & {$[101] 1^{-}$} & -16.952 & {$[101] 1^{-}$} & -13.752 \\
${ }^{15} C$ & ${ }^{12} C$ & 54 & $137(16)$ & 92 & 130 & 35 & 15 & 0.25 & {$[220] 1^{+}$} & -2.611 & {$[101] 3^{-}$} & -19.631 \\
${ }^{17} C$ & ${ }^{12} C$ & 904 & $129(22)$ & 122 & 120 & 31 & 32 & 0.45 & {$[211] 3^{+}$} & -2.705 & {$[101] 3^{-}$} & -21.906 \\
${ }^{19} C$ & ${ }^{12} C$ & 910 & $233(51)$ & 134 & 152 & 31 & 263 & -0.43 & {$[202] 3^{+}$} & -3.316 & {$[101] 1^{-}$} & -27.048 \\
${ }^{8} B$ & ${ }^{12} C$ & 285 & $89(2)$ & 101 & 99 & 34 & 10 & 0.63 & {$[110] 1^{-}$} & -14.054 & {$[101] 3^{-}$} & -1.880 \\
${ }^{12} B$ & ${ }^{12} C$ & 67 & $81(5)$ & 41 & 65 & 45 & 70 & 0.18 & {$[101] 1^{-}$} & -6.212 & {$[101] 3^{-}$} & -15.585 \\
${ }^{13} B$ & ${ }^{12} C$ & 57 & $59(4)$ & 53 & 46 & 44 & 16 & 0.10 & {$[110] 1^{-}$} & -7.378 & {$[101] 3^{-}$} & -18.321 \\
${ }^{14} B$ & ${ }^{12} C$ & 50 & $153(15)$ & 67 & 97 & 32 & 15 & 0.38 & {$[220] 1^{+}$} & -1.992 & {$[101] 3^{-}$} & -18.686 \\
${ }^{15} B$ & ${ }^{12} C$ & 43 & $108(13)$ & 88 & 104 & 68 & 73 & 0.59 & {$[220] 1^{+}$} & -2.611 & {$[101] 3^{-}$} & -19.631 \\
\hline \hline
\end{tabular}

amongst the nuclei investigated here are ${ }^{14} B$ and ${ }^{19} C$ which based on the relatively weak binding of the valence neutrons and measurements of one neutron removal cross-sections have suggested to be one neutron halo systems [23]. The singleparticle energy for proton $\epsilon_{p}$ and neutron $\epsilon_{n}$ for the last occupied orbit are given in the 11th and 13th column of Table 2. The last proton for ${ }^{8} B$ and ${ }^{9} \mathrm{C}$ and the outer most neutron for ${ }^{14,15} B$ and ${ }^{17,19} C$ are loosely bound which are the possible candidates for either proton-halo (or skin) or neutronhalo (skin). Going back to the analysis of Figure 2, which gives us enough indication for the absence of halo-like structure in ${ }^{9,12} \mathrm{C}$. Contrary to the case of ${ }^{9} \mathrm{C}$ and ${ }^{12} \mathrm{C}$ a thin-layer of neutron distribution spread spatially to a large extent in case of ${ }^{19} C$, which looks like a halo-nucleus. This behavior is also reflected in the one neutron removal reaction crosssection. In this particular case of ${ }^{19} \mathrm{C}$, the calculated result $\sigma_{-1 n}(I I)=263 \mathrm{mb}$ is more closure to the experimental value of $\sigma_{-1 n}=233 \pm 51 \mathrm{mb}$ than the $\sigma_{-1 n}$ obtained by using Eqn. (7). If we recall the statement of Abu-Ibrahim et al. [12], then ${ }^{19} \mathrm{C}$ is a halo-nucleus. On the other hand, the measured one neutron removal reaction cross-section of ${ }^{14} B$ is larger than its neighbors suggest the weak binding of the last neutron and extended valence density distribution.

Summarising the whole discussions of Tables 1 and 2, in general, one can say it very briefly that except few cases like ${ }^{12,17} \mathrm{C}+{ }^{12} \mathrm{C}$ and ${ }^{8,13} \mathrm{~B}+{ }^{12} \mathrm{C}$ the spherical density used from RMF (NL3) and E-RMF (G2) fails to reproduce the data. When we use the deformed densities to evaluate the one neutron cross-section, the predicted $\sigma_{-1 n}(I)$ matches reasonably well with the experimental measurement. In this case, only the result of the system ${ }^{19} \mathrm{C}+{ }^{12} \mathrm{C}$ deviate from the observation. However, the results predicted by Eq. (10) disagree largely with the experiments irrespective of the densities used. Complementating to Eq. (7), the $\sigma_{1 n}(I I)$ matches with the lone case ${ }^{19} \mathrm{C}+{ }^{12} \mathrm{C}$ agreeing with the prediction of Ref. [12].

In Figure 3, we have presented the $\sigma_{-1 n}(I)$ with various incident energies for ${ }^{19} C+{ }^{12} C$ using the spherical NL3, NL$\mathrm{SH}$ and $\mathrm{G} 2$ densities in the Glauber model calculation. We also compare our results with the deformed NL-SH densities obtained from the axially deformed RMF. All the spherical densities reproduce similar elastic $\left(\sigma_{-1 n}^{e l}\right)$ and inelastic $\left(\sigma_{-1 n}^{i e l}\right)$ one neutron removal reaction cross-section. The deformed NL-SH densities has a large impact on the evaluation of $\sigma_{-1 n}$ unlike to the total nuclear reaction cross-section $\sigma_{r}$, 


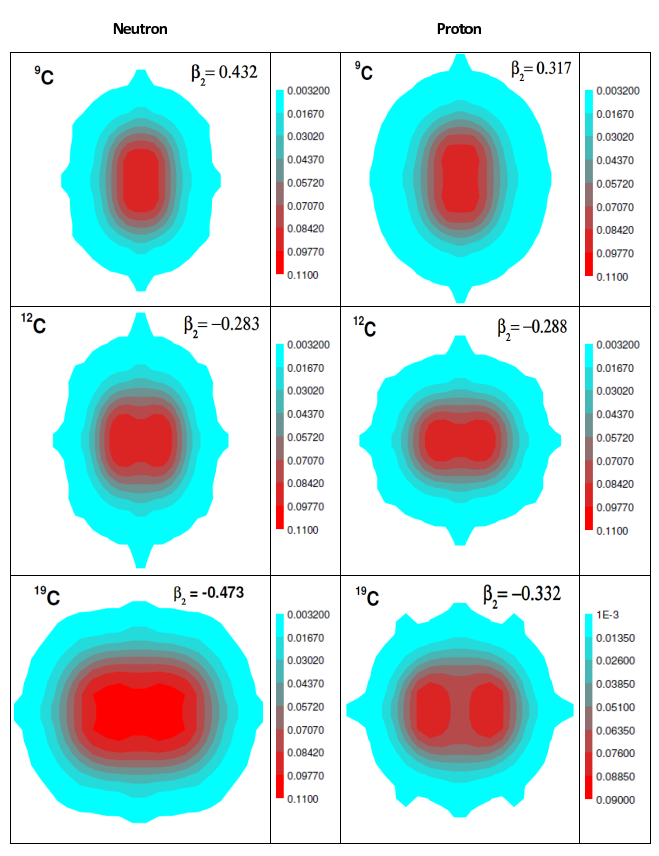

FIG. 2: The axially deformed density distribution for ${ }^{9,12,19} \mathrm{C}$ with RMF (NL-SH) parameter set.

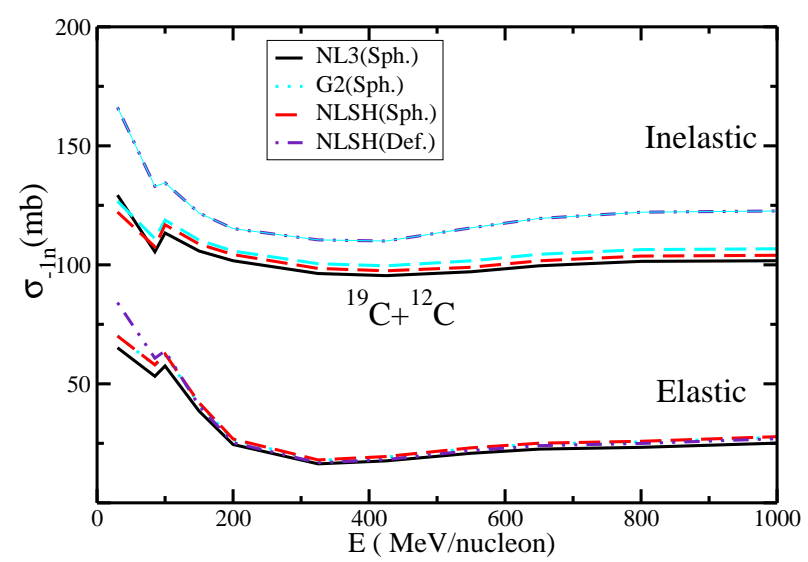

FIG. 3: The energy dependence of the neutron removal cross-section for ${ }^{19} C+{ }^{12} C$ system using spherical densities of RMF (NL3), RMF (NL-SH) and E-RMF (G2) parameter sets for both elastic and inelastic processes. The result obtained by deformed RMF (NL-SH) densities is also given for the comparison. which is evident from Figure 3 and consistent with $\sigma_{-1 n}(I)$. The deformed $\sigma_{-1 n}^{i e l}$ value constantly over-estimate the spherical $\sigma_{-1 n}^{i e l}$ starting from low to very high incident energy of the projectile.

\section{SUMMARY AND CONCLUSION}

In summary, one neutron removal reaction cross-sections for the neutron-rich isotopes have been calculated using the densities obtained from RMF (NL3) and E-RMF (G2) formalisms for spherical and deformed NL-SH parameter sets. The dependence of $\sigma_{-1 n}$ on single-particle energy of the last occupied nucleon is seen in our present calculations. That means, although the total nuclear reaction cross-section does not show a significant difference, the $\sigma_{-1 n}$ values differ from each other depending on the NL3 or G2 parameter set. The $\sigma_{-1 n}$ are in good agreement with the experiments, when we consider the deformation effect in the densities. The Glauber model fails for halo systems and in this case ${ }^{19} \mathrm{C}+{ }^{12} \mathrm{C}$ is a typical example. In such case, the difference between the total reaction cross-section from the consecutive nuclei is applicable to evaluate $\sigma_{-1 n}(I I)$. It is also concluded in the present paper that the deformation effects for one neutron removal crosssection is very much crucial unlike to the total reaction crosssection $\sigma_{r}$. In other words, the Glauber model reproduce the experimental data pretty well while considering the deformed densities for stable nuclei as projectile. On the other hand, when we estimate the difference of reaction cross-section of nuclei with mass number $\mathrm{A}$ and that of A-1 in an isotopic chain, we get good agreement with the experimental data for halo cases.

\section{ACKNOWLEDGMENTS}

We are thankful to Dr. BirBikram Singh and Mr. M. Bhuyan for the fruitful discussions. This work is supported in part by Council of Scientific \& Industrial Research (No.03 (1060) 06/EMR-II), as well as the Department of Science and Technology, Govt. of India, project No. SR/S2/HEP-16/2005.

\section{References}

[1] A. Shukla, B. K. Sharma, R. Chandra, P. Arumugam and S. K. Patra, Phys. Rev. C 76, 034601 (2007); B. K. Sharma, S. K. Patra, Raj K. Gupta, A. Shukla, P. Arumugam, P. D. Stevenson and Greiner Walter, J. Phys. G32, 2089 (2006).

[2] S. K. Patra and R. N. Panda, arXiv:0906.3797; S. K. Patra, R. N. Panda, P. Arumugam and Raj K. Gupta, Phys. Rev. C 80, 064602 (2009).

[3] E. C. Simpson and J. A. Tostevin, Phys. Rev. C 79, 024616 (2009).
[4] S. K. Patra and C. R. Praharaj, Phys. Rev. C 44, 2552 (1991); B. D. Serot and J. D. Walecka, Adv. Nucl. Phys. 16, 1 (1986); P. Ring, Prog. Part. Nucl. Phys. 37, 193 (1996).

[5] G. A. Lalazissis, J. König and P. Ring, Phys. Rev. C 55, 540 (1997).

[6] R. J. Furnstahl, B. D. Serot and H. B. Tang, Nucl. Phys. A615, 441 (1997).

[7] M. Del Estal, M. Centelles, X. Viñas and S. K. Patra, Phys. Rev. C 63, 044321 (2001); S. K. Patra, M. Del Estal, M. Centelles, 
X. Viñas, Phys. Rev. C 63, 024311 (2001); B. D. Serot and J. D. Walecka, Int. J. Mod. Phys. E6, 515 (1997).

[8] R. J. Glauber, Lectures on Theoretical Physics, edited: W. E. Brittin and L. C. Dunham (Interscience, New York), Vol.1, p.315 (1959).

[9] P. J. Karol, Phys. Rev. C 11, 1203 (1975).

[10] J. Chauvin, D. Lubrun, A. Lounis and M. Buenerd, Phys. Rev. C 28, 1970 (1983); M. Buenerd, A. Lounis, J. Chauvin, D. Lebrun, P. Martin, G. Duhamel, J. C. Gondrand and P. D. Saintignon, Nucl. Phys. A 424, 313 (1984).

[11] P. Shukla, Phys. Rev. C 67, 054607 (2003).

[12] B. Abu-Ibrahim, Y. Ogawa, Y. Suzuki and I. Tanihata, comp. Phys. Comm. 151, 369 (2003).

[13] A. Bhagwat and Y. K. Gambhir, Phys. Rev. C 77, 027602 (2008); ibid C 73, 054601 (2006); ibid C 73, 024604 (2006); ibid C 69, 01315 (2004); ibid C 68, 044301 (2003).

[14] S. K. Charagi and S. K. Gupta, Phys. Rev. C 41, 1610 (1990);
S. K. Charagi, Phys. Rev. C 48, 452 (1993); S. K. Charagi and S. K. Gupta, Phys. Rev. C 46, 1982 (1992); S. K. Charagi and S. K. Gupta, Phys. Rev. C 56, 1171 (1997).

[15] Y. K. Gambhir, P. Ring and A. Thimet, Ann. Phys. (N.Y) 198, 132 (1990).

[16] D. Cortina-Gil D et al. 2001 Eur. Phys. J. A10, 49 (2001).

[17] E. Sauvan et al., Phys. Rev. C 69, 044603 (2004); E. Sauvan et al., Phys. Lett. B1, 491 (2000).

[18] Wu Cuie et al., J. Phys. G31, 47 (2005).

[19] D. L. Olson et al., Phys. Rev. C 28, 1602 (1983).

[20] M. M. Sharma, M. A. Nagarajan and P. Ring, Phys. Lett. B312, 377 (1993).

[21] P. -G. Reinhard, Rep. Prog. Phys. 52, 439 (1989).

[22] P. Arumugam, B. K. Sharma, S. K. Patra and Raj K. Gupta, Phys. Rev. C 71, 064308 (2005).

[23] D. Bazin et al., Phys. Rev. C 57, 2156 (1998). 